Article

\title{
The Characterization and Application of Two Liquid Crystal Mixtures in the Low THz Region
}

\author{
Andong Zheng ${ }^{1,2}$, Xiujun Chu ${ }^{1,2}$, Pengjun Wang ${ }^{1,2}$, Peng Wang ${ }^{1,2}$, Sheng Gao ${ }^{1,2}$, \\ Jun Yang ${ }^{1, *} \mathbb{\infty}$, Hongbo Lu ${ }^{1}$, Guangsheng Deng ${ }^{1}\left(\mathbb{D}\right.$ and Zhiping Yin ${ }^{1} \mathbb{C}$ \\ 1 Special Display and Imaging Technology Innovation Center of Anhui Province, \\ Academy of Opto-electric Technology, Hefei University of Technology, Hefei 230009, China; \\ 18656098638@163.com (A.Z.); xjchu@mail.hfut.edu.cn (X.C.); wangpj@mail.hfut.edu.cn (P.W.); \\ wpvilf@126.com (P.W.); gao1sheng001@outlook.com (S.G.); bozhilu@hfut.edu.cn (H.L.); \\ dgsh@hfut.edu.cn (G.D.); zpyin@hfut.edu.cn (Z.Y.) \\ 2 School of Electronic Science and Applied Physcis, Anhui Province, Hefei University of Technology, \\ Hefei 230009, China \\ * Correspondence: junyang@hfut.edu.cn; Tel.: +86-551-6290-2352
}

Received: 14 January 2020; Accepted: 7 February 2020; Published: 9 February 2020

\begin{abstract}
In the previous work, two new nematic liquid crystal (NLC) mixtures, E7-2 and S200-2, were produced by adding eight LC monomers to two commercial LCs S200 and E7, respectively. At $\lambda=589 \mathrm{~nm}$, the birefringence $(\Delta n)$ characteristics of the two LC nematic mixtures E7-2 $(\Delta n=0.260)$ and S200-2 $(\Delta n=0.298)$ are greater than those of the commercial LC E7 $(\Delta n=0.224)$ and S200 $(\Delta n=0.266)$. The properties $\left(T_{\mathrm{N}-\mathrm{I}}, \varepsilon / /, \Delta \epsilon, K_{11}\right.$, and $\left.K_{33}\right)$ of these four NLCs were measured. A double-layer metal loop arrays modulation structure based on metamaterial (MM) metal-dielectric-metal (MDM) was designed and fabricated for use in the THz frequency range. The results show that the LC mixtures E7-2 and S200-2 have greater modulation depth $(M D)$ and less modulation insertion loss (IL) than E7 and S200 at THz frequencies. The results show that LC mixtures have significant potential for designing active tunable LC-based devices in the $\mathrm{THz}$ and visible light range.
\end{abstract}

Keywords: liquid crystals; large birefringence; dielectric anisotropy; modulation

\section{Introduction}

In recent years, terahertz $(\mathrm{THz})$ technologies have been used for $\mathrm{THz}$ wireless communications [1], $\mathrm{THz}$ imaging [2], biomedical research [3], and environmental monitoring [4]. It is critical to control $\mathrm{THz}$ waves, with low-loss and high modulation, for applications using THz systems. Therefore, tunable $\mathrm{THz}$ functional devices have gradually become a major research topic [5]. There are many kinds of modulation mechanisms in the relevant materials that have been reported, such as graphene [6,7], $\mathrm{VO}_{2}$ [8], electron gas [9], and liquid crystals (LCs) [10]. In 2014, the nematic liquid crystal (NLC)-based metamaterial was designed, fabricated, and used in $\mathrm{THz}$ tunable devices [11]. The transmittance tunability of the system is approximately $19 \%$ by changing the magnitude of the AC bias voltage from 0 to 300 V. In 2015, Kowerdziej et al. proposed a tunable LC THz MM device. As the applied voltage increased to $200 \mathrm{~V}$, a maximum tuning value of $22 \%$ occurred at $0.82 \mathrm{THz}$. However, the modulation depth of the LC-based metamaterial is still very narrow and the bias voltage is too high.

Liquid crystals, in which the anisotropy can be changed by electric or magnetic means, have been successful at controlling and propagating electromagnetic waves. Wavelengths have included millimeter waves, visible light, and THz waves [12-14]. High birefringence values are especially important for devices working in the millimeter or $\mathrm{THz}$ ranges of electromagnetic waves. In recent years, 
there has been increasing interest in characterizing LC materials as a tunable materials unit for $\mathrm{THz}$ applications, such as modulators [15], filters [16], phase shifters [17,18], switches [19], and absorbers [20]. High birefringence nematic liquid crystals have been broadly researched to satisfy the needs of the extensive variety of optical infrared range photonics devices [21]. Compared to NLCs for use in the visible region, these NLCs show weak birefringence in $\mathrm{THz}$ regions. Some papers demonstrated that the birefringence exhibited by LCs is relatively low in the THz band $[22,23]$. The relatively low birefringence can lead to performance limitations for $\mathrm{THz}$ electronically tunable devices. An effective way to solve these problems is to synthesize high birefringence LC compound materials. Recently, Vieweg et al. reported the birefringence of the LC mixture BL037, from $\Delta n=0.16$ at $0.5 \mathrm{THz}$ to $\Delta n=0.2$ at $2.5 \mathrm{THz}$ [24]. Wang et al. proposed a new LC mixture, called NJU-LDn-4, with a large birefringence of 0.306 from 0.4 to $1.6 \mathrm{THz}$ [25]. Reuter et al. proposed the high birefringence LC mixtures 1852 $(\Delta n=0.33$ at $0.5 \mathrm{THz})$ and $1825(\Delta n=0.38$ at $0.5 \mathrm{THz})$ [26].

In the previous work, two new NLCs mixtures (E7-2 and S200-2) were proposed [27], which can be used to construct tunable $\mathrm{THz}$ devices due to tunable dielectric properties. In this paper, the more physical properties of these mixture LCs were measured and analyzed. Currently, to develop effective terahertz modulators and switches, electromagnetic metamaterials, an artificial composite material, are essential components. They consist of two- or three-dimensional arrays of tailored metallic unit cells [28]. In order to directly manipulate $\mathrm{THz}$ waves, we demonstrated $\mathrm{THz} \mathrm{LC}$ modulators based on electromagnetic metamaterials and E7-2, S200-2 LC mixtures. The experimental results show that the metrics $\Delta T, M D$, and $I L$ of these mixture-based LCs can be significantly higher than those of commercial LCs in their working frequency band. This suggests that two high birefringence NLCs can also be used to fabricate highly efficient and functional $\mathrm{THz}$ devices, such as phase shifters and switches.

\section{Materials and Methods}

In order to obtain NLCs with high birefringence, eight LC monomers were designed and synthesized based on multi-ring rigid cores and polar terminal groups in Figure 1 [29]. We produced two nematic liquid crystals mixtures, E7-2 and S200-2, by adding eight LC monomers to two commercial NLCs: E7 (Jiangsu Hecheng Technology Co., Ltd. Nanjing, Jiangsu province, China.), and SLC103014-200 (S200) (Shijiangzhuang Chengzhi Yonghua Display Material Co., Ltd. Shijiangzhuang, Hebei province, China.) [27]. The NLC mixture of E7-2 and S200-2 were composed of $84 \mathrm{wt} \%$ of two commercial LC materials (E7 and S200), and $2 \mathrm{wt} \%$ of each new liquid crystal monomer. Dielectric properties of these two LC mixtures were measured using a frequency-selective surface in the sub-THz band. The dielectric anisotropy of the nematic E7-2 and S200-2 LC mixtures was 16.3\% and $16.4 \%$ higher than that of the commercial E7 and S200 LCs, respectively [27].

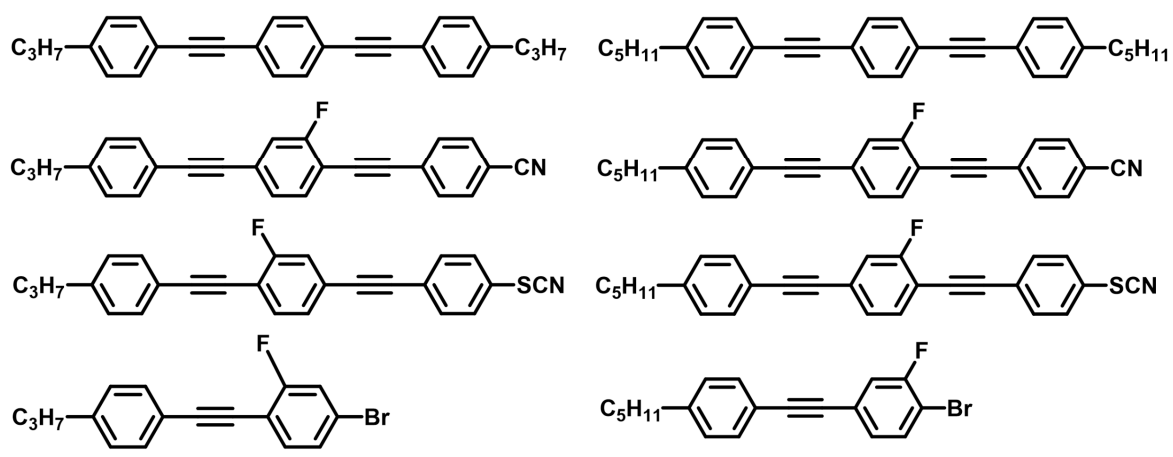

Figure 1. Chemical structures of the eight liquid crystal (LC) monomers.

The physical properties $\left(n_{0}, \Delta n, T_{\mathrm{N}-\mathrm{I}}, \varepsilon_{/ /}, \Delta \epsilon, K_{11}\right.$, and $\left.K_{33}\right)$ of the eutectic mixtures (E7-2 and S200-2) were measured using two commercial LC materials (E7 and S200) as benchmarks for comparison. The measurement results are shown in Table 1. The birefringence of NLC mixtures have been measured using the abbe refractometer (NAR-4T) at $\lambda=589 \mathrm{~nm}$. The measured birefringence of E7 is $\Delta n=0.224$ 
and E7-2 is $\Delta n=0.260$. The birefringence measured of S200 is $\Delta n=0.224$. The S200-2 is $\Delta n=0.260$. The birefringence values of the LC mixtures E7-2 and S200-2 are 16.1\% and 12.0\% higher than the commercial LC materials E7 and S200, respectively. It means that eutectic mixtures with more benzene rings, NCS terminal, and triple bridge bond exhibit larger birefringence values when compared to commercial LCs. The clearing point $\left(T_{\mathrm{N}-\mathrm{I}}\right)$ was measured using differential scanning calorimetry. The values of $T_{\mathrm{N}-\mathrm{I}}$ for $\mathrm{E} 7$ and E7-2 are $58.2{ }^{\circ} \mathrm{C}$ and $72.2{ }^{\circ} \mathrm{C}$, respectively, and the values of $T_{\mathrm{N}-\mathrm{I}}$ for $\mathrm{S} 200$ and S200-2 are $101.1^{\circ} \mathrm{C}$ and $114.9^{\circ} \mathrm{C}$, respectively. The $T_{\mathrm{N}-\mathrm{I}}$ of the eutectic mixtures is much larger than it is for commercial NLCs. This means eutectic mixtures have wide nematic phase temperature ranges. The dielectric properties $\left(\varepsilon_{/ /}\right.$and $\left.\Delta \epsilon\right)$, and the splay $\left(K_{11}\right)$ and bend $\left(K_{33}\right)$ elastic constants of these NLC mixtures, were measured using Instec's automatic liquid crystal test equipment (ITO cell thickness: $11 \mu \mathrm{m})$. Here, $\varepsilon_{/ /}$is the maximum dielectric constant of the LC cell and $\Delta \epsilon\left(\Delta \epsilon=\varepsilon_{/ /}-\varepsilon_{\perp}\right)$ is the LC's dielectric anisotropy at $1 \mathrm{kHz}$. The values of $\varepsilon_{/ /}, \Delta \epsilon, K_{11}$ and $K_{33}$ in eutectic mixtures (E7-2 and S200-2) are smaller than that in commercial LCs E7 and S200 at $1 \mathrm{kHz}$.

Table 1. Physical properties of nematic liquid crystals (NLCs) E7, E7-2, and S200 and S200-2.

\begin{tabular}{cccccccc}
\hline LC & $n_{\mathbf{o}}$ & $\boldsymbol{\Delta} \boldsymbol{n}$ & $\boldsymbol{T}_{\mathbf{N - I}}\left({ }^{\circ} \mathbf{C}\right)$ & $\varepsilon_{/ /}$ & $\boldsymbol{\Delta} \epsilon$ & $\boldsymbol{K}_{\mathbf{1 1}}(\mathbf{P N})$ & $\boldsymbol{K}_{\mathbf{3 3}}(\mathbf{P N})$ \\
\hline E7 & 1.517 & 0.224 & 58.2 & 16.7 & 11.1 & 7.2 & 18.1 \\
E7-2 & 1.522 & 0.260 & 72.2 & 15.4 & 10.1 & 7.1 & 11.3 \\
S200 & 1.515 & 0.266 & 101.1 & 19.8 & 14.9 & 8.4 & 12.3 \\
S200-2 & 1.516 & 0.298 & 114.9 & 16.0 & 13.0 & 7.1 & 3.9 \\
\hline
\end{tabular}

\section{Applications of Devices for Terahertz Continuous Wave Modulation}

The application frequency range of interest for a LC for a tunable metamaterial-LC-metamaterial (MLM) structure is about from $290 \mathrm{GHz}$ to $320 \mathrm{GHz}$. The unit cell structure of the LC-based tunable THz MLM is described in Figure 2a. The double-layered metal MM structure is a periodic surface with identical two-dimensional arrays of sub-wavelength circular air loops, using the ultraviolet lithography process shown in Figure 2b. This structure was embedded in the LC layer contained within an MLM structure, as is shown in Figure 2. It is composed of two parallel fused silica substrates that are separated by a $45 \mu \mathrm{m}$ spacer. A layer of arrayed metal metamaterial unit cells was deposited on the inner surface of the quartz substrate. Polyimide (Pi) is an organic insulating material with a low dielectric constant. It has been widely used to align LC molecules. Both electrodes are spin-coated with sulfonic Pi as the alignment layers and are photo-aligned. A cell is infiltrated with the NLCs. The optical axis of the LC is initially parallel to the connecting wires. These LC materials are composed of anisotropic molecules whose direction may change due to an external electrical field between the copper patterned layer and the metal ground.

The tunable THz MLM with $33 \times 33$ arrays of sub-wavelength circular air loops was manufactured on a quartz substrate. The upper and the lower substrates are a pair of $220 \mu \mathrm{m}$ thick quartz glass plates. The MLM geometry parameters are as follows: $\mathrm{p}=449 \mu \mathrm{m}, \mathrm{r}_{1}=182 \mu \mathrm{m}, \mathrm{r}_{2}=206 \mu \mathrm{m}, \mathrm{w}=16 \mu \mathrm{m}$, $\mathrm{h}_{1}=\mathrm{h}_{2}=220 \mu \mathrm{m}, \mathrm{h}_{\mathrm{lc}}=45 \mu \mathrm{m}$, and $\mathrm{h}_{3}=500 \mathrm{~nm}$.

The cell is driven by applying a $1 \mathrm{kHz}$ square wave current signal through copper tapes connecting the electrodes to the electric power source. As the applied voltage increased, the optical axes of the LC molecules gradually change along the electric field vector as shown in Figure 2c. Without an electric field, the long axes of the LC molecules lie parallel to the surface of each quartz plate. The dielectric constant of the LC layer is $\varepsilon_{\perp}$. When a saturating bias voltage is applied to the LC layer, the long axes of the LC molecules will be parallel to the electric field. The dielectric constant of the LC layer is $\varepsilon / /$. The structure demonstrates a pronounced radiant mode that can be used to develop THz emitters. 


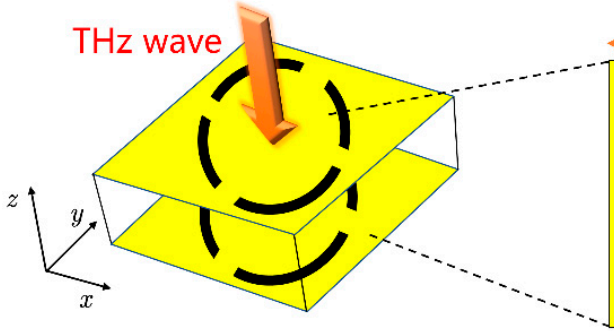

a

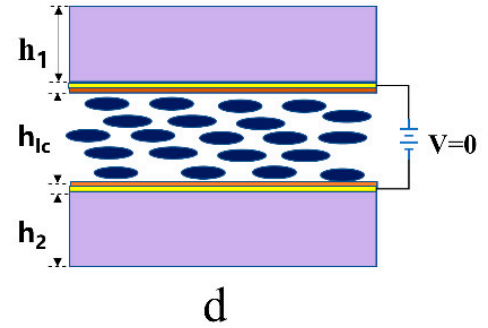

$\mathbf{P}$

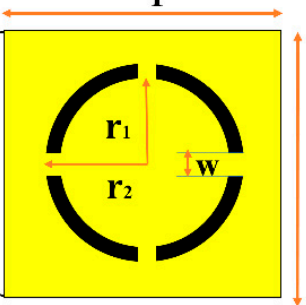

b

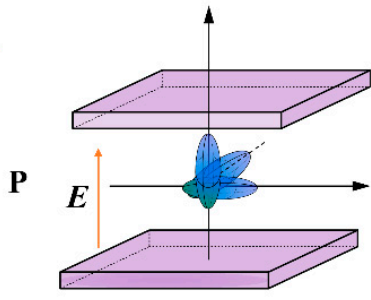

$\mathrm{C}$

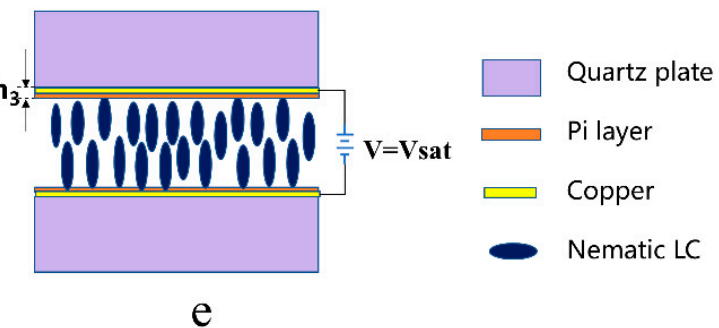

e

Figure 2. (a) The unit cell structure. (b) Layout of the unit cell structure of the copper pattern. (c) Orientation of the LC molecules with different electric fields. (d,e) Side view orientation of the LC molecules for biased and unbiased states.

The transmittance spectrum can be changed to different NLC dielectric constants. The transmission characteristics and the LC parameters are calculated and analyzed for $\mathrm{THz}$ electromagnetic waves in the frequency range from 220 to $330 \mathrm{GHz}$. The EM performance of the LC-based MLM structure was analyzed and optimized using the finite difference time domain (FDTD) method. In the simulation, assuming that the unit cell is located in a periodic environment, and illuminated by a linear polarization and normal incidence plane wave. Period boundary conditions are used in the $x$-axis and $y$-axis directions, and the boundary condition in the $z$-axis direction is set to open. So, the EM properties of the $\mathrm{THz}$ MDM structure were obtained by FDTD. The simulated transmittance spectra of the modulator for different dielectric constants is shown in Figure 3.

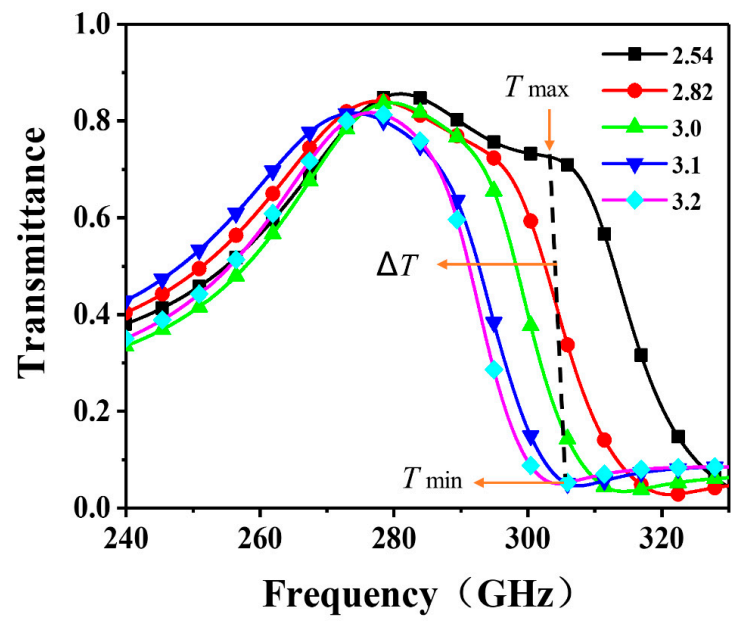

Figure 3. Simulated transmittance spectra for different dielectric constants.

Numerical results for resonant frequencies were obtained from the numerical FDTD, and fitted to the measured results. The conductivity of the copper layer was set to $5.8 \times 10^{7} \mathrm{~S} / \mathrm{m}$. The dielectric constants are set from 2.54 to 3.2. The transmittance spectrum can be changed to different LC dielectric constants. As the dielectric constants increase, the lower transmittance spectrum will shift toward a 
low resonance frequency. This reveals that the LC-based MLM structure can change from its maximum to its minimum transmittance value.

The modulation depth $(M D)$, insertion loss $(I L)$, and $\Delta T$ are used to describe the performance of the LC-based modulator device. The $M D, I L$, and $\Delta T$ of the modulator structure at a frequency point can be calculated using:

$$
\begin{gathered}
\Delta T=T_{\max }-T_{\min }, \\
M D=\Delta T / T_{\max }, \\
I L=-10 \lg \left(T_{\max }\right), \\
\Delta \varphi=\varphi_{\max }-\varphi_{\min },
\end{gathered}
$$

where the parameters $T_{\max }$ and $T_{\min }$ are maximum and minimum transmittances of the structure at a certain frequency point. The variables $\varphi_{\max }$ and $\varphi_{\min }$ are the maximum and minimum phases of the structure at a certain frequency point, respectively.

\section{Experimental Results and Discussions}

In order to experimentally verify the performance of the proposed device, the MLM structure was fabricated with a two-layer metal MM array using the ultraviolet lithography process. The manufactured device and the microscopic image of the unit cell are shown in Figures $3 \mathrm{~b}$ and $4 \mathrm{a}$. The EM characteristics of the MLM structure were measured at room temperature using a vector network analyzer system and the transmission spectra were obtained at different bias voltages as shown in Figure 4c. The effective dielectric constant of the LCs molecules depends on the bias voltage. The Agilent N5224A vector network analyzer and the VDI mm-wave module extender (from $220 \mathrm{GHz}$ to $325 \mathrm{GHz}$ ) were used to measure the transmittance spectra of the LC samples. Two pairs of lenses were used to focus the $\mathrm{THz}$ wave. The maximum voltage $(20 \mathrm{~V})$ with a $1 \mathrm{kHz}$ square wave was applied to the patterned copper layer and the copper ground plane in the experiment.

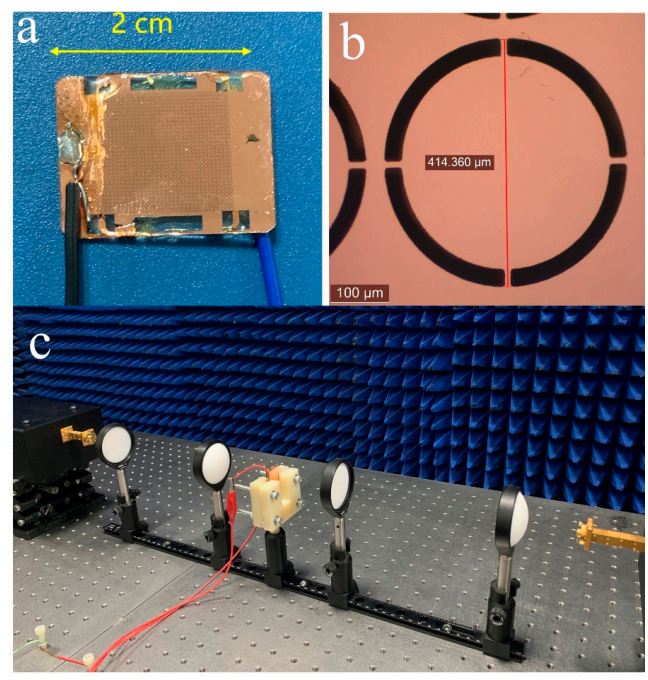

Figure 4. The photograph of sample and schematics of the experimental setup. (a) Fabricated sample structure $\left(20 \times 20 \mathrm{~mm}^{2}\right)$. (b) A polarized microscope image of a single unit cell. (c) Experimental measurement setup.

For applied voltages increasing from $0 \mathrm{~V}$ to $20 \mathrm{~V}$, the transmission characteristics of the transmissive MLM structure are shown in Figures 4 and 5, respectively. When the bias voltage is varied from 0 to $4 \mathrm{~V}$, samples have rapid spectral transmittance changes. The transmittance of the samples slowly rises and eventually stabilizes when the bias voltage is higher than $4 \mathrm{~V}$. For a zero applied bias voltage, the black curve $(0 \mathrm{~V})$ represents the maximum transmission spectrum. When the applied voltage increased to 
its maximum, the pink curve $(20 \mathrm{~V})$ is the minimum transmission spectra. The $\Delta T$ and $M D$ values increase as the external applied bias is increased at a frequency point.

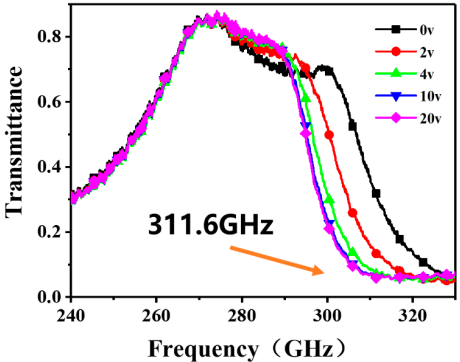

a

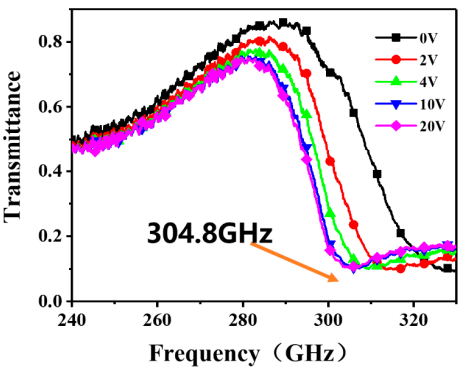

C

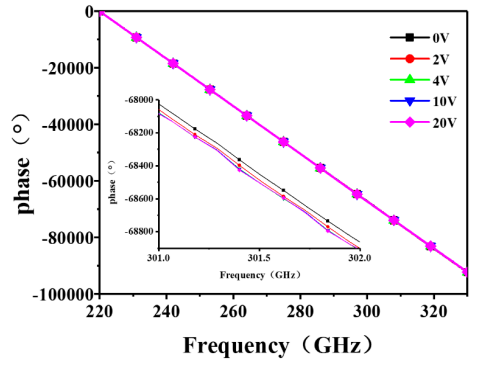

b

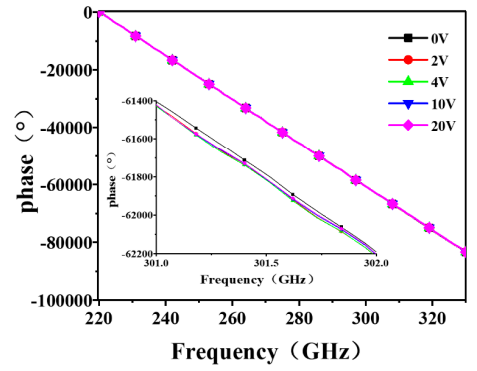

d

Figure 5. (a) Transmission spectra of the LC E7 sample. (b) Phase information of the LC E7 sample. (c) Transmission spectra of the LC E7-2 sample. (d) Phase information about the LC E7-2 sample.

To further investigate the performance of the demonstrated sample at multiple frequency points, from Figure 5, we extracted the $M D, I L, \Delta T$, and $\Delta \varphi$ for specific frequency points. Table 2 lists the values for from frequencies ranging from $296 \mathrm{GHz}$ to $312 \mathrm{GHz}$.

Table 2. Comparison of two LC samples (E7 and E7-2) at certain frequency points.

\begin{tabular}{ccccc}
\hline Frequency $(\mathrm{GHz})$ & $\boldsymbol{\Delta}$ & $\boldsymbol{M} \boldsymbol{D}$ & $\boldsymbol{I L}(\mathrm{dB})$ & $\boldsymbol{\Delta} \boldsymbol{\varphi}\left({ }^{\circ}\right)$ \\
\hline 296 & $0.27<0.42$ & $0.39<0.53$ & $1.65>1.01$ & $69.1: 46.5$ \\
297 & $0.35<0.45$ & $0.50<0.58$ & $1.50>1.15$ & $54.9: 46.5$ \\
300 & $0.47<0.56$ & $0.69<0.76$ & $1.54>1.51$ & $59.5: 25.5$ \\
303 & $0.49<0.55$ & $0.78<0.83$ & $1.94>1.70$ & $46.0:-4.7$ \\
305 & $0.45<0.51$ & $0.81<0.84$ & $2.52>2.08$ & $14.8:-10.8$ \\
306 & $0.44<0.48$ & $0.83>0.81$ & $0.26>0.29$ & $24.2:-34.3$ \\
307 & $0.40<0.43$ & $0.82>0.79$ & $0.31>2.69$ & $1.70:-39.5$ \\
312 & $0.23>0.25$ & $0.77>0.61$ & $5.38>4.50$ & $-42.0:-80.0$ \\
\hline
\end{tabular}

Compared to the resonance frequency point $(311.6 \mathrm{GHz})$ of the low transmittance spectrum of the E7 LC characteristic shown in Figure 5a, the resonance frequency point of the E7-2 shift toward 304.8 GHz in Figure 5b, and the $\Delta T$ and $M D$ values of the LC E7-2 are larger than that of the commercial LC E7 between $296 \mathrm{GHz}$ and $305 \mathrm{GHz}$. The IL of the LC E7-2 sample is less than that of the commercial LC E7 from $296 \mathrm{GHz}$ to $312 \mathrm{GHz}$. For example, the values of $\Delta T$ and $M D$ of the LC E7-2 are 0.56 and 0.76 at $300 \mathrm{GHz}$, respectively, and $\Delta T$ and $M D$ of the LC E7 are 0.47 and 0.69 , respectively. The value of $I L$ $(1.51 \mathrm{~dB})$ of the LC E7-2 sample is less than the IL $(1.54 \mathrm{~dB})$ of the commercial LC E7. These comparisons show that the dielectric anisotropy of mixture LC E7-2 is higher than that of commercial LC E7. The results indicate that mixture LC E7-2 can significantly increase the maximum transmission spectra of double-layer metal loop arrays' modulation. The relative phases of E7 and E7-2 have a small change $\left(\Delta \varphi<90^{\circ}\right)$ at different voltages. 
Figure 6 shows the transmission spectra results of NLC S200 and S200-2. These transmission spectra curves were measured for different voltages. The values of $M D, I L, \Delta T$, and $\Delta \varphi$ are listed in Table 3.

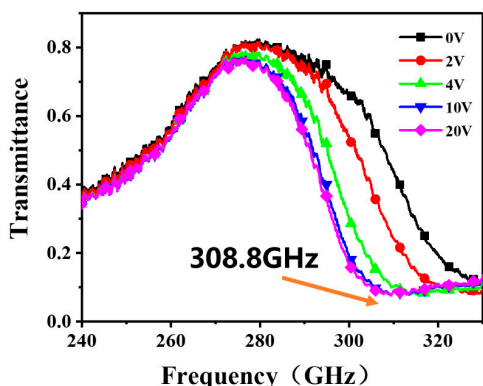

a

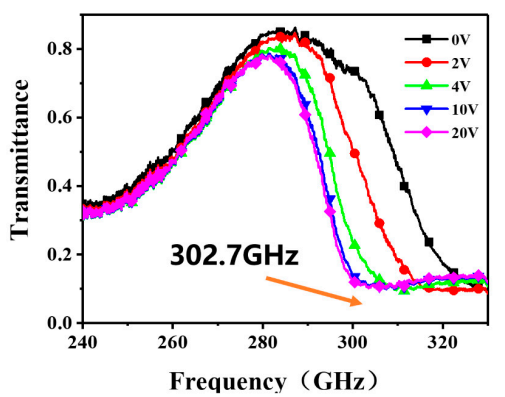

C

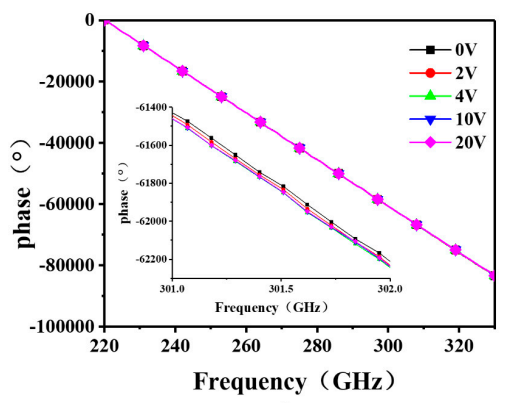

b

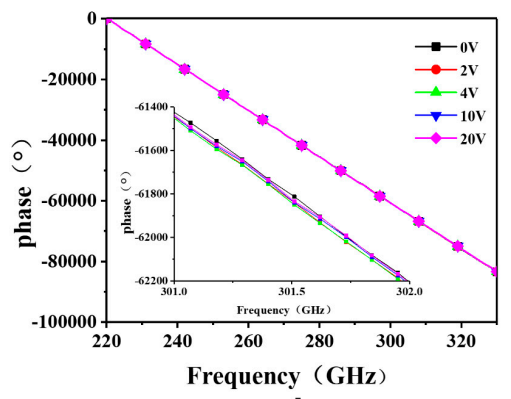

d

Figure 6. (a) LC S200 sample transmission spectra. (b) LC S200 sample phase information. (c) LC S200-2 sample transmission spectra. (d) LC S200-2 sample phase information.

Table 3. Comparison of two LC samples (S200 and S200-2) at certain frequency points.

\begin{tabular}{ccccc}
\hline Frequency $(\mathrm{GHz})$ & $\boldsymbol{\Delta}$ & $\boldsymbol{M} \boldsymbol{D}$ & $\boldsymbol{I L}(\mathrm{dB})$ & $\boldsymbol{\Delta} \boldsymbol{\varphi}\left({ }^{\circ}\right)$ \\
\hline 296 & $0.40<0.52$ & $0.56<0.67$ & $1.94>1.20$ & $59.6: 39.2$ \\
297 & $0.42<0.55$ & $0.61<0.72$ & $2.05>1.23$ & $40.8: 36.1$ \\
300 & $0.47<0.56$ & $0.69<0.76$ & $1.54>1.51$ & $30.7: 20.8$ \\
303 & $0.49<0.55$ & $0.78<0.83$ & $1.94>1.70$ & $2.2:-10.7$ \\
305 & $0.45<0.51$ & $0.81<0.84$ & $2.52>2.08$ & $19.3:-16.9$ \\
306 & $0.44<0.48$ & $0.83>0.81$ & $0.26>0.29$ & $-10.6:-31.3$ \\
310 & $0.40<0.43$ & $0.82 .>0.79$ & $0.31>2.69$ & $-74.3:-82.5$ \\
312 & $0.23>0.25$ & $0.77>0.61$ & $5.38>4.50$ & $-56.8:-65.4$ \\
\hline
\end{tabular}

Compared to the resonance frequency point $(308.8 \mathrm{GHz})$ of the low transmittance spectrum (the pink curve) of the S200 in Figure 6a, the resonant frequency point of the S200-2 shifts toward 302.7 GHz in Figure 6b. The $\Delta T$ and $M D$ of the LC S200-2 mixture are larger than those of the commercial LC S200 from $296 \mathrm{GHz}$ to $305 \mathrm{GHz}$. The IL of the LC S200-2 sample is less than that of the commercial LC S200. The phase of the S200 and S200-2 have small changes $\left(\Delta \varphi<90^{\circ}\right)$ at different voltages in the working frequency band.

For example, the $\Delta T(0.6)$ and $M D(0.83)$ of S200-2 are larger than the corresponding $\Delta T(0.49)$ and $M D(0.75)$ values of the commercial LC S200 at $300 \mathrm{GHz}$. The IL (1.36 dB) of the LC S200-2 sample is less than the IL ( $2.2 \mathrm{~dB})$ of the commercial LC S200 at $300 \mathrm{GHz}$. According to numerical FDTD simulation data, the values show that the dielectric anisotropy of the LC S200-2 mixture is higher than that of commercial LC S200.

These results indicate that the LC mixtures E7-2 and S200-2 can significantly increase the maximum transmission spectra of double-layer metal loop arrays' modulation capabilities. The dielectric anisotropy of LC mixtures can be significantly increased by adding eight LC monomers. 
The birefringence these NLC materials exhibit high into the visible range would present comparatively high dielectric anisotropy in $\mathrm{THz}$ range as well.

\section{Conclusions}

In this paper, two new NLC mixtures (the LC S200-2 and the LC E7-2) were proposed and the birefringence of the nematic LC E7-2 and S200-2 mixtures is higher by $16.1 \%$ and $12.0 \%$ than the birefringence of the commercial LC products E7 and S200 at visible light, respectively. The eutectic mixtures (E7-2 and S200-2) have wide nematic phase temperature ranges. The values of $\varepsilon_{\| /}, \Delta \epsilon, K_{11}$, and $K_{33}$ in eutectic mixtures are smaller than those of the commercial LCs E7 and S200 at $1 \mathrm{kHz}$. Based on the proposed LC and metamaterials, a THz MLM structure was fabricated to control terahertz waves using the ultraviolet lithography process. The experimental results are shown that LC mixtures E7-2 and S200-2 have larger $\Delta T, M D$, and less IL for modulation than those E7 and S200 at THz frequencies for normal incidence of $\mathrm{THz}$ waves. The relative phases of $\mathrm{LC}$ mixtures have small change $\left(\Delta \varphi<90^{\circ}\right)$ at different voltages. It also means that the $\Delta \epsilon$ of the commercial liquid crystals (E7 and S200) is improved by adding eight LC monomers. Our work may provide an effective solution for the design of new high birefringence $\mathrm{LC}$ materials in the $\mathrm{THz}$ range.

Author Contributions: Original draft, fabricated, A.Z. and X.C.; designed, P.W. (Peng Wang); measurement, P.W. (Pengjun Wang) and S.G.; review and editing, J.Y.; resources, J.Y. and H.L.; methodology, G.D.; conceptualization, Z.Y. All authors have read and agreed to the published version of the manuscript.

Funding: This research is supported by the National Natural Science Foundation of China (Grant No. 61871171).

Conflicts of Interest: The authors declare no conflict of interest.

\section{References}

1. Boulogeorgos, A.A.A.; Alexiou, A.; Merkle, T.; Schubert, C.; Elschner, R.; Katsiotis, A.; Stavrianos, P.; Kritharidis, D.; Chartsias, P.K.; Kokkoniemi, J.; et al. Terahertz Technologies to Deliver Optical Network Quality of Experience in Wireless Systems Beyond 5G. IEEE Commun. Mag. 2018, 56, 144-151. [CrossRef]

2. Guerboukha, H.; Nallappan, K.; Skorobogatiy, M. Toward real-time terahertz imaging. Adv. Opt. Photonics 2018, 10, 843-938. [CrossRef]

3. Yang, X.; Zhao, X.; Yang, K.; Liu, Y.P.; Liu, Y.; Fu, W.L.; Luo, Y. Biomedical Applications of Terahertz Spectroscopy and Imaging. Trends Biotechnol. 2016, 34, 810-824. [CrossRef] [PubMed]

4. Liu, Y.; Liu, H.; Tang, M.Q.; Huang, J.Q.; Liu, W.; Dong, J.Y.; Chen, X.P.; Fu, W.L.; Zhang, Y. The medical application of terahertz technology in non-invasive detection of cells and tissues: Opportunities and challenges. RSC Adv. 2019, 9, 9354-9363. [CrossRef]

5. Tian, L.L.; Chu, F.; Dou, H.; Li, L.; Wang, Q.H. Electrically Tunable-Focusing Liquid Crystal Microlens Array with Simple Electrode. Crystals 2019, 9, 431. [CrossRef]

6. Wei, M.Q.; Zhang, D.N.; Li, Y.P.; Zhang, L.; Jin, L.C.; Wen, T.L.; Bai, F.M.; Zhang, H.W. High-Performance All-Optical Terahertz Modulator Based on Graphene/TiO2/Si Trilayer Heterojunctions. Nanoscale Res. Lett. 2019, 14, 1-6. [CrossRef]

7. Wang, G.; Zhang, X.; Wei, X.; Zhang, G. Tunable and Polarization-Independent Plasmon-Induced Transparency in a Fourfold Symmetric Metal-Graphene Terahertz Metamaterial. Crystals 2019, 9, 632. [CrossRef]

8. Zhou, G.C.; Dai, P.H.; Wu, J.B.; Jin, B.B.; Wen, Q.Y.; Zhu, G.H.; Shen, Z.; Zhang, C.H.; Kang, L.; Xu, W.W.; et al. Broadband and high modulation-depth $\mathrm{THz}$ modulator using low bias controlled VO2-integrated metasurface. Opt. Express 2017, 25, 17322-17328. [CrossRef]

9. Zhao, Y.C.; Wang, L.; Zhang, Y.X.; Qiao, S.; Liang, S.X.; Zhou, T.C.; Zhang, X.L.; Guo, X.Q.; Feng, Z.H.; Lan, F.; et al. High-Speed Efficient Terahertz Modulation Based on Tunable Collective-Individual State Conversion within an Active $3 \mathrm{~nm}$ Two-Dimensional Electron Gas Metasurface. Nano Lett. 2019, 19, 7588-7597. [CrossRef]

10. Shen, Z.X.; Zhou, S.H.; Ge, S.J.; Duan, W.; Ma, L.L.; Lu, Y.Q.; Hu, W. Liquid crystal tunable terahertz lens with spin-selected focusing property. Opt. Express 2019, 27, 8800-8807. [CrossRef] 
11. Kowerdziej, R.; Olifierczuk, M.; Parka, J.; Wrobel, J. Terahertz characterization of tunable metamaterial based on electrically controlled nematic liquid crystal. Appl. Phys. Lett. 2014, 105, 022908. [CrossRef]

12. Kowerdziej, R.; Jaroszewicz, L.; Olifierczuk, M.; Parka, J. Experimental study on terahertz metamaterial embedded in nematic liquid crystal. Appl. Phys. Lett. 2015, 106, 092905. [CrossRef]

13. Li, J.; Chu, D. Liquid Crystal-Based Enclosed Coplanar Waveguide Phase Shifter for 54-66 GHz Applications. Crystals 2019, 9, 650. [CrossRef]

14. Duan, W.; Chen, P.; Ge, S.J.; Liang, X.; Hu, W. A Fast-Response and Helicity-Dependent Lens Enabled by Micro-Patterned Dual-Frequency Liquid Crystals. Crystals 2019, 9, 111. [CrossRef]

15. Wang, J.; Tian, H.; Wang, Y.; Li, X.Y.; Cao, Y.J.; Li, L.; Liu, J.L.; Zhou, Z.X. Liquid crystal terahertz modulator with plasmon-induced transparency metamaterial. Opt. Express 2018, 26, 5769-5776. [CrossRef]

16. Yan, D.X.; Li, J.S.; Jin, L.F. Light-controlled tunable terahertz filters based on photoresponsive liquid crystals. Laser Phys. 2019, 29, 025401. [CrossRef]

17. Yang, J.; Xia, T.Y.; Jing, S.C.; Deng, G.S.; Lu, H.B.; Fang, Y.; Yin, Z.P. Electrically Tunable Reflective Terahertz Phase Shifter Based on Liquid Crystal. J. Infrared Millim. Terahertz Waves 2018, 39, 439-446. [CrossRef]

18. Du, Y.; Tian, H.; Cui, X.; Wang, H.; Zhou, Z.X. Electrically tunable liquid crystal terahertz phase shifter driven by transparent polymer electrodes. J. Mater. Chem. C 2016, 4, 4138-4142. [CrossRef]

19. Yuan, Y.H.; He, J.A.; Liu, J.S.; Yao, J.Q. Electrically controlled broadband THz switch based on liquid-crystal-filled multi-layer metallic grating structures. J. Phys. Conf. Ser. 2011, 276. [CrossRef]

20. Wang, R.X.; Li, L.; Liu, J.L.; Yan, F.; Tian, F.J.; Tian, H.; Zhang, J.Z.; Sun, W.M. Triple-band tunable perfect terahertz metamaterial absorber with liquid crystal. Opt. Express 2017, 25, 32280-32289. [CrossRef]

21. Ge, S.J.; Chen, P.; Shen, Z.X.; Sun, W.F.; Wang, X.K.; Hu, W.; Zhang, Y.; Lu, Y.Q. Terahertz vortex beam generator based on a photopatterned large birefringence liquid crystal. Opt. Express 2017, 25, 12349-12356. [CrossRef] [PubMed]

22. Li, X.F.; Tan, N.; Pivnenko, M.; Sibik, J.; Zeitler, J.A.; Chu, D.P. High-birefringence nematic liquid crystal for broadband THz applications. Liq. Cryst. 2016, 43, 955-962. [CrossRef]

23. Vieweg, N.; Jansen, C.; Shakfa, M.K.; Scheller, M.; Krumbholz, N.; Wilk, R.; Mikulics, M.; Koch, M. Molecular properties of liquid crystals in the terahertz frequency range. Opt. Express 2010, 18, 6097-6107. [CrossRef] [PubMed]

24. Vieweg, N.; Shakfa, M.K.; Koch, M. BL037: A nematic mixture with high terahertz birefringence. Opt. Commun. 2011, 284, 1887-1889. [CrossRef]

25. Wang, L.; Lin, X.W.; Liang, X.; Wu, J.B.; Hu, W.; Zheng, Z.G.; Jin, B.B.; Qin, Y.Q.; Lu, Y.Q. Large birefringence liquid crystal material in terahertz range. Opt. Mater. Express 2012, 2, 1314-1319. [CrossRef]

26. Reuter, M.; Vieweg, N.; Fischer, B.M.; Mikulicz, M.; Koch, M.; Garbat, K.; Dąbrowski, R. Highly birefringent, low-loss liquid crystals for terahertz applications. APL Mater. 2013, 1, 012107. [CrossRef]

27. Zheng, A.D.; Xia, X.; Gao, S.; Yang, J.; Lu, H.B.; Deng, G.S.; Yin, Z.P. Dielectric properties of two high birefringence liquid crystal mixtures in the Sub-THz band. Liq. Cryst. 2019, 47. [CrossRef]

28. Yang, J.; Wang, P.; Shi, T.; Gao, S.; Lu, H.B.; Yin, Z.P.; Lai, W.E.; Deng, G.S. Electrically tunable liquid crystal terahertz device based on double-layer plasmonic metamaterial. Opt. Express 2019, 27, 27039-27045. [CrossRef]

29. Lapanik, V.; Sasnouski, G.; Timofeev, S.; Shepeleva, E.; Evtyushkin, G.; Haase, W. New highly anisotropic liquid crystal materials for high-frequency applications. Liq. Cryst. 2018, 45, 1242-1249. [CrossRef]

(C) 2020 by the authors. Licensee MDPI, Basel, Switzerland. This article is an open access article distributed under the terms and conditions of the Creative Commons Attribution (CC BY) license (http://creativecommons.org/licenses/by/4.0/). 